\title{
Erratum to "On a Classical Theorem on the Diameter and Minimum Degree of a Graph"
}

\author{
Verónica HERNÁNDEZ Domingo PESTANA José M. RODRÍGUEZ \\ Departamento de Matemáticas, Universidad Carlos III de Madrid, Avenida de la Universidad 30, \\ 28911 Leganés, Madrid, Spain \\ E-mail:vehernan@math.uc3m.es_dompes@math.uc3m.es_jomaro@math.uc3m.es
}

\begin{abstract}
The original version of the article was published in [1]. Unfortunately, the original version of this article contains a mistake: in Theorem 6.2 appears that $\beta(n, \Delta)=(n-\Delta+5) / 4$ but the correct statement is $\beta(n, \Delta)=(n-\Delta+4) / 4$. In this erratum we correct the theorem and give the correct proof.
\end{abstract}

Keywords Extremal problems on graphs, diameter, minimum degree, maximum degree, Gromov hyperbolicity, hyperbolicity constant, finite graphs

MR(2010) Subject Classification $05 \mathrm{C} 75,05 \mathrm{C} 12,05 \mathrm{~A} 20,05 \mathrm{C} 80$

\section{Computation of $\beta(n, \Delta)$}

Lemma 6.1 For all $\Delta \geq 6$ and $n \geq \Delta+1$, we have $\beta(n, \Delta) \leq(n-\Delta+4) / 4$.

Proof Seeking for a contradiction assume that $\beta(n, \Delta)>(n-\Delta+4) / 4$. Thus, there exists $G \in \mathcal{J}(n, \Delta)$ such that $\delta(G)>(n-\Delta+4) / 4$. By Theorem 3.5, $\delta(G) \geq(n-\Delta+5) / 4$. By Theorem 3.6 there exist a geodesic triangle $T=\{x, y, z\}$ with $x, y, z \in J(G)$ and $p \in[x y]$ such that $\delta(G)=d(p,[x z] \cup[y z])$. Since $L(T) \geq 4 \delta(G)$, we have $L(T)=n-\Delta+t$ with $t \geq 5$, and $|V(G) \backslash T|=\Delta-t$.

Fix $v \in V(G)$ with $\operatorname{deg}(v)=\Delta$. We consider now several cases:

Case (A) Assume first that $v \notin T$. Since $|V(G) \backslash T|=\Delta-t$ and $v \in V(G) \backslash T$, we have $|N(v) \cap T| \geq t+1$. Define $t_{1}=|N(v) \cap[x y]|$ and $t_{2}=|N(v) \cap(T \backslash[x y])|$. Thus, $t_{1}+t_{2} \geq t+1$. Since $[x y]$ is a geodesic, we have $0 \leq t_{1} \leq 3$ and, therefore, $t_{2} \geq t-2 \geq 3$ and $N(v) \cap(T \backslash[x y]) \neq \emptyset$.

Case (A.1) If $t_{1} \geq 2$, then let us choose $\alpha_{x}, \alpha_{y} \in V(G) \cap[x y]$ and $\beta_{x}, \beta_{y} \in V(G) \cap(T \backslash[x y])$, with

$$
\begin{aligned}
& d_{G}\left(x, \alpha_{x}\right)=\min \left\{d_{G}(x, w) \mid w \in N(v) \cap[x y]\right\}, \\
& d_{G}\left(y, \alpha_{y}\right)=\min \left\{d_{G}(y, w) \mid w \in N(v) \cap[x y]\right\}, \\
& d_{[x z] \cup[z y]}\left(x, \beta_{x}\right)=\min \left\{d_{[x z] \cup[z y]}(x, w) \mid w \in N(v) \cap(T \backslash[x y])\right\}, \\
& d_{[x z] \cup[z y]}\left(y, \beta_{y}\right)=\min \left\{d_{[x z] \cup[z y]}(y, w) \mid w \in N(v) \cap(T \backslash[x y])\right\} .
\end{aligned}
$$

Received January 31, 2018, accepted May 16, 2018

Supported by two grants from Ministerio de Economía y Competitividad, Spain (Grant Nos. MTM2013-46374-P and MTM2015-69323-REDT) 
We have $L([x y]) \geq d_{G}\left(x, \alpha_{x}\right)+t_{1}-1+d_{G}\left(\alpha_{y}, y\right)$ and $L([x z] \cup[z y]) \geq d_{[x z] \cup[z y]}\left(x, \beta_{x}\right)+t_{2}-$ $1+d_{[x z] \cup[z y]}\left(\beta_{y}, y\right)$. We can assume that $p \in\left[\alpha_{y} y\right]$, since otherwise the argument is similar (if $p \in\left[x \alpha_{x}\right]$ the argument is symmetric, if $p \in\left[\alpha_{x} \alpha_{y}\right]$ the argument is similar and simpler). Since there exists $v^{\prime} \in N(v) \cap(T \backslash[x y])$, we have $L\left(\left[\alpha_{y} y\right] \cup\left[\alpha_{y}, v\right] \cup\left[v, v^{\prime}\right]\right) \geq 2 \delta(G)$ and, consequently, $d_{G}\left(\alpha_{y}, y\right) \geq 2 \delta(G)-2$. Thus, $L([x y]) \geq d_{G}\left(x, \alpha_{x}\right)+t_{1}-1+2 \delta(G)-2$. We also have

$2 \delta(G) \leq d_{G}(x, y) \leq d_{G}\left(x, \beta_{x}\right)+d_{G}\left(\beta_{x}, v\right)+d_{G}\left(v, \beta_{y}\right)+d_{G}\left(\beta_{y}, y\right)=d_{G}\left(x, \beta_{x}\right)+d_{G}\left(\beta_{y}, y\right)+2$,

$L([x z] \cup[z y]) \geq 2 \delta(G)-2+t_{2}-1$,

$n-\Delta+t=L(T) \geq d_{G}\left(x, \alpha_{x}\right)+t_{1}+t_{2}-6+4 \delta(G) \geq d_{G}\left(x, \alpha_{x}\right)+t-5+n-\Delta+5$,

which is a contradiction if $d_{G}\left(x, \alpha_{x}\right)>0$, i.e., $x \notin N(v)$.

If $x \in N(v)$, then $L([x z] \cup[z y]) \geq t_{2}+d_{[x z] \cup[z y]}\left(\beta_{y}, y\right)$, and the previous argument gives

$$
n-\Delta+t=L(T) \geq t_{1}+t_{2}-5+4 \delta(G) \geq t-4+n-\Delta+5,
$$

which is a contradiction.

Case (A.2) If $t_{1}<2$, then $t_{2} \geq t$. The argument in (A.1) also gives $L([x z] \cup[z y]) \geq$ $2 \delta(G)+t_{2}-3$ in this case. Since $L([x y]) \geq 2 \delta(G)$, we have

$$
n-\Delta+t=L(T) \geq 4 \delta(G)-3+t \geq n-\Delta+5-3+t,
$$

a contradiction.

Case (B) Now, assume that $v \in T$. Since $|V(G) \backslash T|=\Delta-t$, we have $|N(v) \cap T| \geq t$.

Case (B.1) Assume that $v \in[x y]$. Since $[x y]$ is a geodesic and $v \in[x y]$, we have $\mid N(v) \cap$ $[x y] \mid \leq 2$ and, therefore, $|N(v) \cap(T \backslash[x y])| \geq t-2$ and $N(v) \cap(T \backslash[x y]) \neq \emptyset$. Let us choose $\beta_{x}, \beta_{y} \in V(G) \cap(T \backslash[x y])$ as in case (A.1).

We can assume that $p \in[v y]$, since otherwise the argument is similar. We have

$$
\begin{aligned}
& 2 \delta(G) \leq d_{G}(x, y) \leq d_{G}(x, v)+d_{G}\left(v, \beta_{y}\right)+d_{G}\left(\beta_{y}, y\right)=d_{G}(x, v)+d_{G}\left(\beta_{y}, y\right)+1, \\
& L([x z] \cup[z y]) \geq d_{G}\left(x, \beta_{x}\right)+d_{G}\left(\beta_{x}, \beta_{y}\right)+d_{G}\left(\beta_{y}, y\right) \geq 1 / 2+d_{G}\left(\beta_{y}, y\right)+t-3, \\
& L([x y]) \geq d_{G}(x, v)+d_{G}(v, y) \geq d_{G}(x, v)+2 \delta(G)-1, \\
& n-\Delta+t=L(T) \geq d_{G}(x, v)+2 \delta(G)-1+1 / 2+d_{G}\left(\beta_{y}, y\right)+t-3 \\
& \quad \geq 4 \delta(G)+1 / 2+t-5 \geq n-\Delta+t+1 / 2,
\end{aligned}
$$

which is a contradiction.

Case (B.2) Assume that $v \in T \backslash[x y]$. Define $t_{1}=|N(v) \cap[x y]|$ and $t_{2}=|N(v) \cap(T \backslash[x y])|$. Thus, $t_{1}+t_{2} \geq t$. Since $[x y]$ is a geodesic, we have $0 \leq t_{1} \leq 3$ and, therefore, $t_{2} \geq t-3 \geq 2$ and $N(v) \cap(T \backslash[x y]) \neq \emptyset$.

Case (B.2.1) If $t_{1} \geq 2$, then let us choose $\alpha_{x}, \alpha_{y} \in V(G) \cap[x y]$ and $\beta_{x}, \beta_{y} \in V(G) \cap(T \backslash[x y])$ as in case (A.1).

We have $L([x y]) \geq d_{G}\left(x, \alpha_{x}\right)+t_{1}-1+d_{G}\left(\alpha_{y}, y\right)$ and $L([x z] \cup[z y]) \geq d_{[x z] \cup[z y]}\left(x, \beta_{x}\right)+$ $t_{2}-1+d_{[x z] \cup[z y]}\left(\beta_{y}, y\right)$. We can assume that $p \in\left[\alpha_{y} y\right]$, since otherwise the argument is similar. Since $L\left(\left[\alpha_{y} y\right] \cup\left[\alpha_{y}, v\right]\right) \geq 2 \delta(G)$, we have $d_{G}\left(\alpha_{y}, y\right) \geq 2 \delta(G)-1$. Thus, $L([x y]) \geq$ $d_{G}\left(x, \alpha_{x}\right)+t_{1}-1+2 \delta(G)-1$. We also have

$2 \delta(G) \leq d_{G}(x, y) \leq d_{G}\left(x, \beta_{x}\right)+d_{G}\left(\beta_{x}, v\right)+d_{G}\left(v, \beta_{y}\right)+d_{G}\left(\beta_{y}, y\right)=d_{G}\left(x, \beta_{x}\right)+d_{G}\left(\beta_{y}, y\right)+2$, 
$L([x z] \cup[z y]) \geq 2 \delta(G)-2+t_{2}-1$,

$n-\Delta+t=L(T) \geq d_{G}\left(x, \alpha_{x}\right)+t_{1}+t_{2}-5+4 \delta(G) \geq d_{G}\left(x, \alpha_{x}\right)+t-5+n-\Delta+5$,

which is a contradiction if $d_{G}\left(x, \alpha_{x}\right)>0$, i.e., $x \notin N(v)$.

If $x \in N(v)$, then $L([x z] \cup[z y]) \geq t_{2}+d_{[x z] \cup[z y]}\left(\beta_{y}, y\right)$, and the previous argument gives

$$
n-\Delta+t=L(T) \geq t_{1}+t_{2}-4+4 \delta(G) \geq t-4+n-\Delta+5,
$$

a contradiction.

Case (B.2.2) If $t_{1}<2$, then $t_{2} \geq t-1$. The argument in (B.2.1) also gives $L([x z] \cup[z y])$ $\geq 2 \delta(G)+t_{2}-3$ in this case. Since $L([x y]) \geq 2 \delta(G)$, we have

$$
n-\Delta+t=L(T) \geq 4 \delta(G)+t-4 \geq n-\Delta+5+t-4,
$$

which is a contradiction.

Hence, $\beta(n, \Delta) \leq(n-\Delta+4) / 4$.

Theorem 6.2 Consider any $1 \leq \Delta \leq n-1$.

- If $\Delta=1$, then $n=2$ and $\beta(2,1)=0$.

- If $2 \leq \Delta \leq 4$, then $\beta(n, \Delta)=n / 4$.

- If $\Delta \geq 5$, then $\beta(n, \Delta)=(n-\Delta+4) / 4$.

Proof For every $n$ and $\Delta$, Theorem 4.7 gives $\beta(n, \Delta) \leq n / 4$.

If $\Delta=1$ and $G \in \mathcal{J}(n, 1)$, then $G$ is isomorphic to the path graph $P_{2}$. Thus, $n=2$ and $\beta(2,1)=0$.

If $\Delta=2$, then every graph $G \in \mathcal{J}(n, 2)$ is isomorphic to either the path graph $P_{n}$ (if $\delta=1$ ) or the cycle graph $C_{n}$ (if $\delta=2$ ). Since $\delta\left(C_{n}\right)=n / 4$, we conclude $\beta(n, \Delta)=n / 4$.

If $\Delta=3$ or $\Delta=4$, then [43, Proposition 29 and Theorem 30] provide graphs $G_{n, \Delta} \in \mathcal{J}(n, \Delta)$ with $\delta\left(G_{n, \Delta}\right)=n / 4$, which implies $\beta(n, \Delta)=n / 4$.

Assume $\Delta=5$ (thus $n \geq 6$ ). Note that [43, Proposition 29 and Theorem 30] give $\beta(n, 5)<$ $n / 4$, and Theorem 3.5 gives $\beta(n, 5) \leq(n-1) / 4$. Since $\beta(n, 4)=n / 4$ for every $n \geq 5$, there exists a graph $F_{n} \in \mathcal{J}(n-1,4)$ with $\delta\left(F_{n}\right)=(n-1) / 4$ and $w \in V\left(F_{n}\right)$ such that $\operatorname{deg} w=4$ for each $n \geq 6$. Consider a graph $\Gamma$ isomorphic to $P_{2}$ and fix a vertex $v \in V(\Gamma)$. Identify $v$ and $w$ in a single vertex $v^{*}$. We obtain in this way a graph $G_{n} \in \mathcal{J}(n, 5)$ from $F_{n}$ and $\Gamma$, since $\Delta=\operatorname{deg} v^{*}=4+1$. Furthermore, $\left\{F_{n}, \Gamma\right\}$ is the biconnected decomposition of $G_{n}$ and Theorem 3.1 gives $\delta\left(G_{n}\right)=\delta\left(F_{n}\right)=(n-1) / 4$. Therefore, $\beta(n, 5) \geq \delta\left(G_{n}\right)=(n-1) / 4$, and we conclude $\beta(n, 5)=(n-1) / 4$.

Assume now $\Delta \geq 6$. Since $n-\Delta \geq 1$ we can consider a graph $G_{1}$ isomorphic to the cycle graph $C_{n-\Delta+5}$. Consider two points $x, y \in G_{1}$, with $x \in V\left(G_{1}\right)$ and $d_{G_{1}}(x, y)=(n-\Delta+4) / 2$. Denote by $\Gamma_{1}, \Gamma_{2}$ the geodesics in $G_{1}$ joining $x$ and $y$ with $G_{1}=\Gamma_{1} \cup \Gamma_{2}$. Denote by $v_{i}^{j}$ the vertex in $\Gamma_{i}$ with $d_{G_{1}}\left(v_{i}^{j}, x\right)=j$, for $i=1,2$ and $1 \leq j \leq(n-\Delta+5) / 2$. Note that $(n-\Delta+5) / 2 \geq 3$.

Consider a graph $G_{2}$ isomorphic to the star graph with $\Delta+1$ vertices $S_{\Delta} \in \mathcal{J}(\Delta+1, \Delta)$. Denote by $v^{*} \in V\left(G_{2}\right)$ the vertex of maximum degree in $G_{2}$, that is, $\operatorname{deg} v^{*}=\Delta$. Since $\Delta \geq 6$, we can choose vertices $w_{j} \in V\left(G_{2}\right) \backslash\left\{v^{*}\right\}(j=1, \ldots, 6)$.

Identify $x$ and $w_{6}$ in a single vertex $w^{*}$. For each $1 \leq j \leq 2$, identify $v_{1}^{j} \in V\left(\Gamma_{1}\right)$ and $w_{j} \in V\left(G_{2}\right)$ in a single vertex $v_{j}^{*}$, and for each $1 \leq j \leq 3$, identify $v_{2}^{j} \in V\left(\Gamma_{2}\right)$ and $w_{j+2} \in V\left(G_{2}\right)$ 
in a single vertex $v_{j+2}^{*}$. We obtain in this way a graph $G \in \mathcal{J}(n, \Delta)$ from $G_{1}$ and $G_{2}$, since $|V(G)|=n-\Delta+5+\Delta+1-6=n$ and $\Delta=\operatorname{deg} v^{*}$.

Consider the geodesic triangle $T=\{x, y, z\}$ in $G$ with $z=v_{4}^{*}, \Gamma_{1}=[x y]$ and $\Gamma_{2}=[x z] \cup[z y]$. If we consider the midpoint $p$ of $\Gamma_{1}$, then

$$
\beta(n, \Delta) \geq \delta(G) \geq d_{G}\left(p, \Gamma_{2}\right)=d_{G}(p,\{x, y\})=\frac{1}{2} L\left(\Gamma_{1}\right)=\frac{n-\Delta+4}{4} .
$$

Since Lemma 6.1 implies $\beta(n, \Delta) \leq(n-\Delta+4) / 4$, we conclude $\beta(n, \Delta)=(n-\Delta+4) / 4$.

\section{References}

[1] Hernández, V., Pestana, D., Rodríguez, J.: On a classical theorem on the diameter and minimum degree of a graph. Acta Mathematica Sinica, English Series, 33(11), 1477-1503 (2017) 